\title{
Intraoperative left ventricular perforation with false aneurysm formation
}

\author{
G. P. Sharratt, J. K. Ross, J. L. Monro, and A. M. Johnson \\ From the Wessex Regional Cardiothoracic Centre
}

Two cases of perforation of the left ventricle during mitral valve replacement are described. In the first case there was perforation at the site of papillary muscle excision and this was recognized and successfully treated. However, a true ventricular aneurysm developed at the repair site. One month after operation rupture of the left ventricle occurred at a second and separate site on the posterior aspect of the atrioventricular ring. This resulted in a false aneurysm which produced a pansystolic murmur mimicking mitral regurgitation. Both the true and the false aneurysm were successfully repaired. In the second case perforation occurred on the posterior aspect of the atrioventricular ring and was successfully repaired. However, a false ventricular aneurysm developed and ruptured into the left atrium producing severe, but silent, mitral regurgitation. This was recognized and successfully repaired. The implications of these cases are discussed.

Left ventricular perforation during mitral valve replacement is an uncommon event but when it occurs it is often lethal (Roberts and Morrow, 1967; MacVaugh, Joyner, and Johnson, 1971). It can occur at the posterior aspect of the atrioventricular groove or at that portion of the left ventricle which overlies the papillary muscles (Treasure, Rainer, and Sadler, 1974). False ventricular aneurysm formation is a rare complication of myocardial infarction (Gueron et al., 1974), nonpenetrating chest wall trauma (O'Reilly, Kazenelson, and Spellberg, 1970), left ventricular incision (Kerr, Wilcken, and Steiner, 1961; Wychulis et al., 1971), and of a left ventricular diverticulum (Layton and Hately, 1975). Spellberg and O'Reilly (1972) reported 2 cases of false ventricular aneurysm that followed mitral valve replacement and were thought to be related to the mobilization of the left ventricle from dense pericardial adhesions. We report 2 cases in which ventricular perforation occurred during mitral valve replacement and was later complicated by false aneurysm formation. This is out of a total of 343 patients undergoing mitral valve replacement at this centre over the past $3 \frac{1}{2}$ years.

Case 1

\section{Case reports}

A 60-year-old woman presented with a mildly Received 14 June 1976. regurgitant, severely stenosed, and heavily calcified mitral valve. There was minimal aortic regurgitation and the left ventricular cavity was of normal size.

At operation (22 November 1974) the calcification was found not to involve the mitral annulus and there was only moderate disease of the subvalvar mechanism. A $27 \mathrm{~mm}$ Björk-Shiley prosthesis was inserted using multiple interrupted sutures. While attempting to come off bypass the pericardium was found to be filling with blood. Bypass was restarted and a tear was found in the posterior left ventricular wall where the papillary muscle had been removed. The left anterior descending artery and branches of the circumflex coronary artery on either side of the defect were intact. The tear was closed using 'teflon' reinforced mattress sutures and a second layer of continuous sutures. The closure appeared to be effective and bypass was terminated successfully. A haematoma was, however, noted in the atrioventricular groove.

Recovery was uneventful and no murmurs were detected. The electrocardiogram, previously normal apart from mitral $P$ waves, now showed right axis deviation and a broad prominent $R$ wave in lead V1 (Fig. 1A and B). This was thought to indicate a strictly posterior myocardial infarction. Progress, however, was uneventful and she was discharged well 14 days after the operation.

Two weeks later she suddenly developed severe, 
A

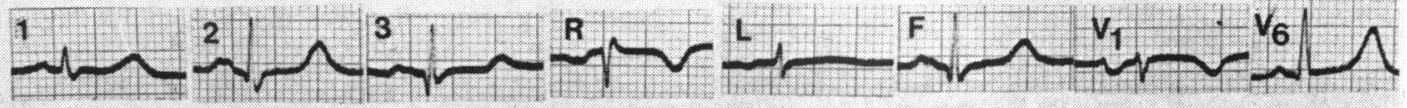

B

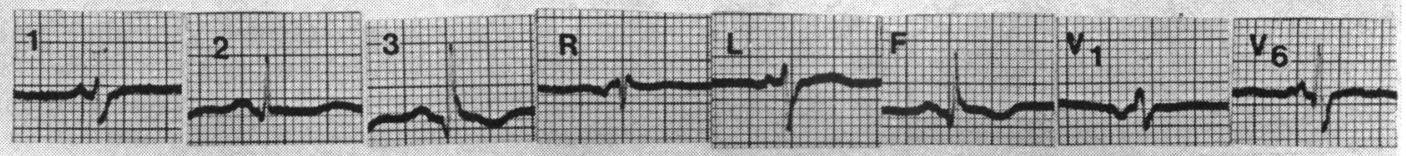

C

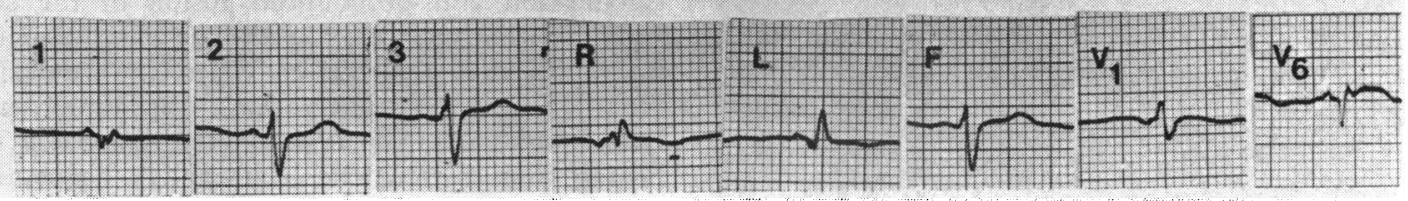

FIG. 1 Electrocardiograms of Case 1: (A) before mitral valve replacement; (B) after mitral valve replacement; and (C) after repair of aneurysms. All traces were recorded at conventional paper speed $(25 \mathrm{~mm} / \mathrm{s})$ and standardization $(1 \mathrm{~cm}=1 \mathrm{mV})$.

gripping, central chest pain and lost consciousness for a few minutes. When she recovered the pain was still present in the central chest and radiated to the left arm. On examination she was cold and sweating, with a blood pressure of $90 / 50 \mathrm{mmHg}$. There was an increased left ventricular impulse and an apical pansystolic murmur (grade 2/4) radiating to the axilla. The electrocardiogram showed no evidence of any further myocardial infarction. The chest $x$-ray film, when compared with the earlier postoperative $x$-ray films, showed the development of a

bulge of the left cardiac border (Fig. 2). The cardiac output rapidly improved and left ventriculography was performed. This showed minimal mitral regurgitation and a small true ventricular aneurysm on the posterolateral aspect of the left ventricle. There was also delayed filling of a false ventricular aneurysm which was situated higher up the posterolateral aspect of the left ventricle. It appeared to fill from the region of the atrioventricular groove.

It was decided to defer operation for repair of

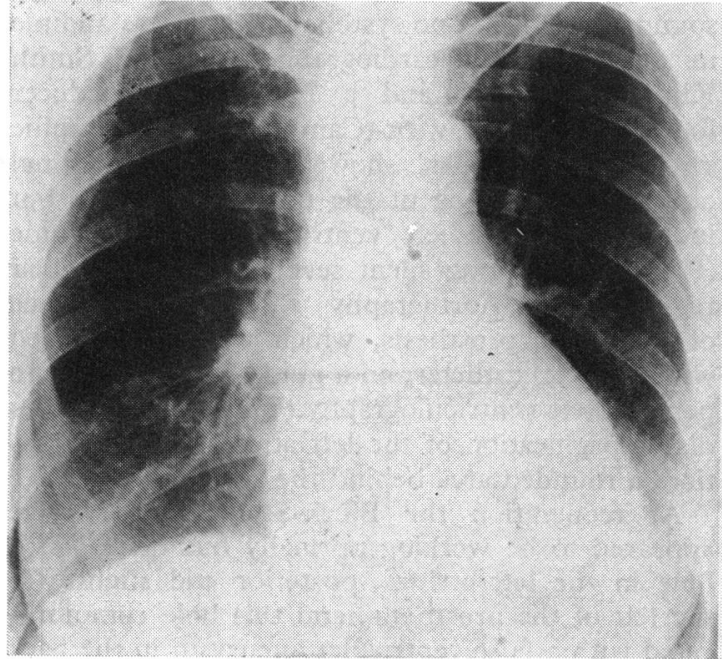

(A)

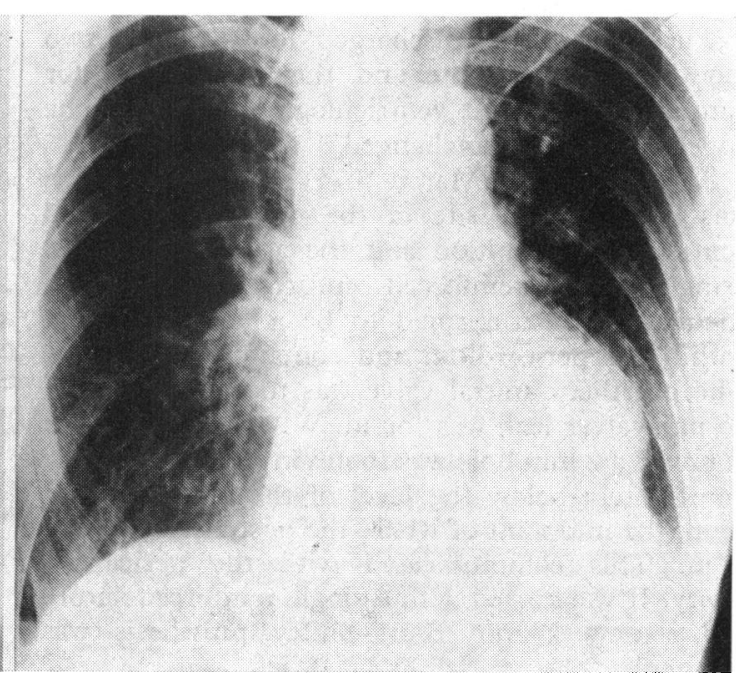

(B)

FIG. 2 (A) Posteroanterior chest $\mathrm{x}$-ray film 10 days after mitral valve replacement in Case 1. (B) Posteroanterior chest $\mathrm{x}$-ray film taken after the episode of severe chest pain in Case 1, showing the development of a bulge on the left cardiac border. 


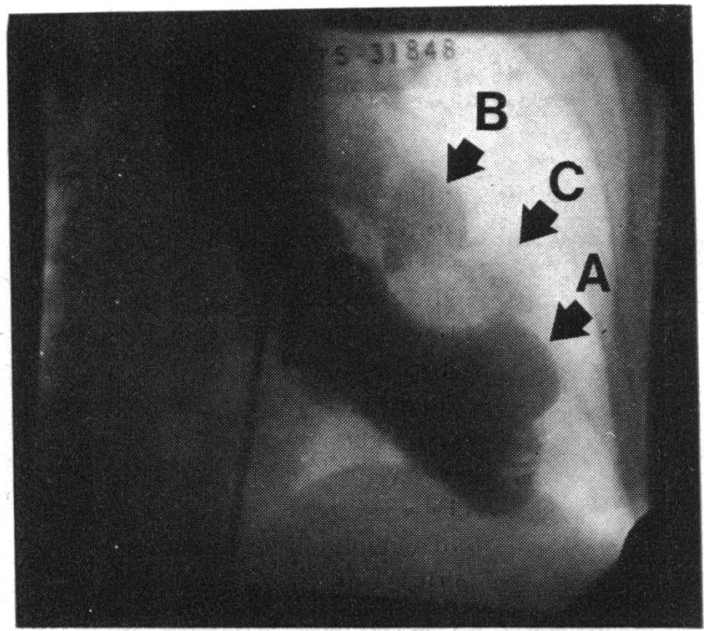

FIG. 3 Left ventriculogram, in the anteroposterior projection, showing a true ventricular aneurysm $(A)$ and a bilocular false ventricular aneurysm ( $B$ and $C$ ) in Case 1.

these aneurysms because the tissues would be friable at this stage. She was, therefore, rested for one month after which left ventriculography was repeated. The true ventricular aneurysm was unchanged but the false aneurysm appeared to have extended to become bilocular (Fig. 3). Systolic jets from the posterior aspect of the atrioventricular groove into the false aneurysm were seen with each ventricular systole.

The patient was discharged for a further two months' rest at home and then readmitted for surgery. A third left ventriculography showed the appearances to be unchanged.

At operation (15 March 1975) the true aneurysm was found at the site of the previously sutured ventricular perforation and the area was plicated using 'tefion' reinforced sutures. The second aneurysm was confirmed to be a false aneurysm within the pericardium and containing thrombus. The prosthetic mitral valve was then exposed and no paravalvar leak was found. When the valve was removed a $2 \mathrm{~mm}$ hole was found in the left ventricle immediately below the level of the mitral ring at about the midpoint of where the posterior cusp had been. This communicated with the pericardial cavity. It was closed with a single reinforced suture and a new $27 \mathrm{~mm}$ Björk-Shiley prosthesis was inserted.

Recovery was again uneventful and there was no longer an apical systolic murmur. The electrocardiogram however showed pathological $Q$ waves in leads I and AVL and left anterior hemiblock, in addition to the abnormal $R$ wave in V1 previously noted (Fig. 1C). She was discharged 15 days after the operation and has remained well.

\section{Case 2}

A 62-year-old woman presented with mixed mitral and aortic valve disease. The valves were not calcified and the left ventricular cavity was moderately enlarged.

At operation (22 September 1975) slight mitral calcification and pronounced fusion of the subvalvar mechanism were found. The mitral and aortic valves were replaced by Starr-Edwards valves (size $4 \mathrm{M}$ in the mitral position). Cardiopulmonary bypass was ended but it was noted that blood was oozing from behind the heart and a haematoma was found in the posterior atrioventricular groove. Cardiopulmonary bypass was restarted. The mitral prosthesis was removed and a tear in the posterior atrioventricular groove was identified. This was repaired using 'teflon' reinforced mattress sutures and a $31 \mathrm{~mm}$ Björk-Shiley valve was then inserted into the mitral position. Uneventful recovery followed and there was no evidence of myocardial infarction.

Three months later she was readmitted with a month's history of severe dyspnoea. On examination she was in congestive heart failure and had an increased left ventricular impulse and pronounced left parasternal lift. The opening click of the Björk-Shiley mitral prosthesis was audible and there was a loud third heart sound. The StarrEdwards aortic prosthesis made multiple opening sounds (Fig. 4) but no systolic murmur was audible in any area. Echocardiography, using a Smith Kline Ekoline 20 and a $2 \cdot 25 \mathrm{MHz}$ transducer focused at $5 \mathrm{~cm}$, with Cambridge photographic strip chart recorder, showed apparently normal opening and closing of the mitral prosthesis, but indicated a large left ventricular stroke volume (Fig. 4), suggesting silent severe mitral paravalvar regurgitation. Aortography showed competence of the aortic prosthesis, which was then crossed with a pigtail catheter, on a guide-wire, in order to perform left ventriculography. This showed immediate opacification of the left atrium and, in addition, a rounded area behind the aorta (Fig. 5).

At reoperation the Björk-Shiley mitral valve appeared to be working normally but there was a hole in the left atrium, posterior and slightly to the left of the prosthesis, and this hole communicated with a false ventricular aneurysm in the back of the left ventricular wall.

The valve was excised and this showed the opening into the false aneurysm. The hole in the left atrium and the false aneurysm were closed. A new 


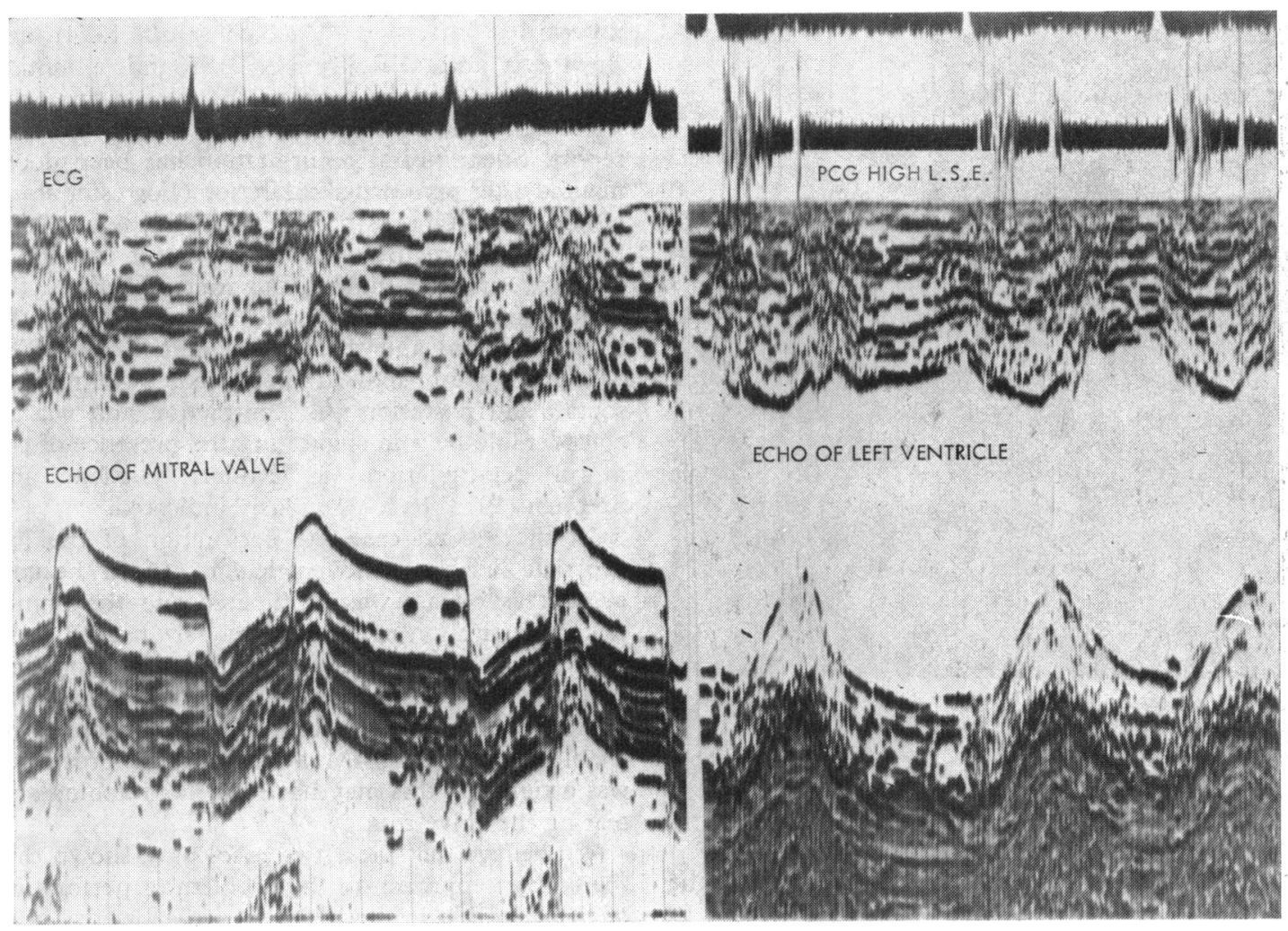

FIG. 4 Echocardiogram of Björk-Shiley prosthesis in the mitral position in Case 2 showing normal opening and closing movement. Echocardiogram of the left ventricle in Case 2, showing pronounced synchronous, posterior wall and septal contraction. The phonocardiogram (PCG) shows multiple sounds caused by opening of the Starr-Edwards aortic prosthetic valve.

$25 \mathrm{~mm}$ Björk-Shiley prosthesis was then inserted and bypass was terminated. Recovery was uneventful and the electrocardiogram: showed no evidence of myocardial infarction. She was discharged two weeks later with no evidence of cardiac failure.

\section{Discussion}

Perforation of the ventricular wall in the region of the papillary muscles may occur during removal of the mitral valve (Treasure et al., 1974; Zacharias et al., 1975) particularly if this is being done under conditions of ischaemic arrest with a flaccid heart. Traction on the valve mechanism, to expose the papillary muscles for their division, causes inward tenting of the ventricular wall. Transection of the papillary muscle base then 'button-holes' the ventricular wall.

Treasure et al. (1974) state that perforation at the posterior aspect of the atrioventricular groove tends to occur when there is extensive calcification of the mitral annulus, when the cavity of the left ventricle is small, and when there has been previous surgery. None of these factors, however, was present in the two cases we report.

Rupture at either site may become evident immediately or there may be a delay of a few hours while the damaged tissue breaks down and then ruptures. There is often, therefore, no opportunity for the surgeon to try to repair the rupture, and even if the patient can be returned to the operating theatre an impossible situation may be found. Attempted repair of either variety of perforation carries with it grave danger of damage to adjacent coronary vessels, but this is particularly so with rupture into the atrioventricular groove. Here the pad of fat in the groove becomes infiltrated and swollen with blood and the overlying epicardial layer ruptures at multiple points so that the exact position of the underlying tear is impossible to locate. Deep sutures placed so as to reattach atrium to ventricle have to pass beneath both the circumflex artery and the coronary sinus, and either or both may be 


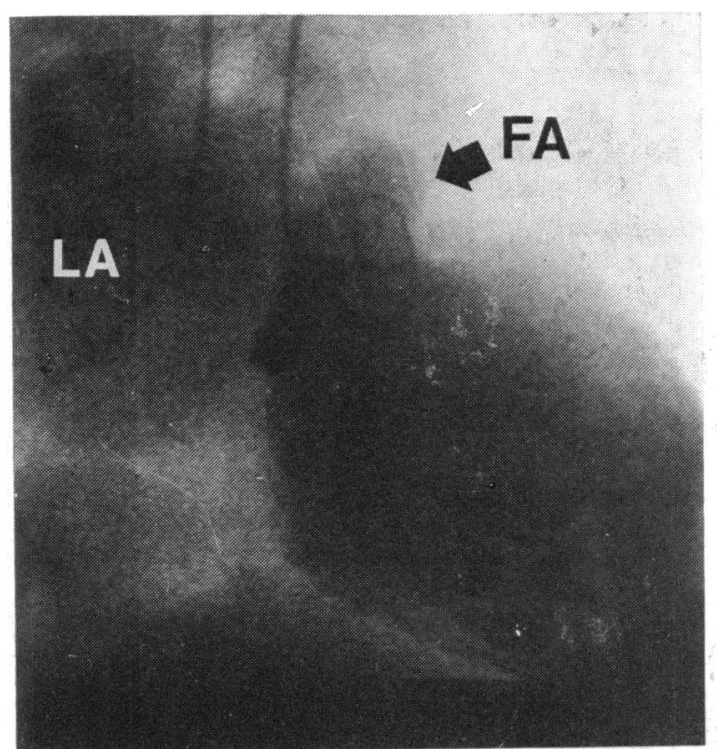

FIG. 5 Left ventriculogram in a right anterior oblique projection. Contrast medium is shown to be filling the left atrium ( $L A$ ) and there is an opacity behind the aortic root resulting from the false aneurysm (FA).

compromised in the process. In a review of the published material Zacharias et al. (1975) found 19 cases of which 13 had been fatal.

Case 1 had rupture of the left ventricle at both sites. The perforation resulting from papillary muscle resection was evident before bypass had been completely terminated, and this probably accounts for the successful outcome. The rupture at the atrioventricular groove in this case was unusual in that it occurred one month after the operation and was small. It was probably caused by tissue necrosis around a single suture which had been placed too deeply. Because of pericardial adhesions this was not immediately fatal but resulted in the formation of a false ventricular aneurysm. This type of ventricular aneurysm tends to rupture (Vlodaver, Coe, and Edwards, 1975), but repair was electively delayed and left ventriculography was performed three times in the patient and appeared to be a safe procedure. It is thought that the delay allowed in this patient before further operation added considerably to the safety of the repair.

It is of interest that the pansystolic murmur in this patient appears to have been produced by blood jetting into the false aneurysm. Mitral regurgitation was of the trivial amount normal for a Björk-Shiley prosthesis and no paravalvar leak was present. Such a murmur has been reported in association with a false aneurysm occurring after myocardial infarction (MacNeil et al., 1974), and Spellberg and O'Reilly (1972) found a similar murmur in the two cases of false ventricular aneurysm after mitral valve replacement that they report. Silent mitral regurgitation has been documented after myocardial infarction (Forrester et al., 1971) and with prosthetic valves (Sanders, 1969). Silent mitral regurgitation was present in Case 2 and was unusual in that the route taken by the regurgitant jet was from the left ventricle to the false aneurysm and then to the left atrium. The echocardiogram showed increased synchronous, septal, and posterior wall contraction and was of considerable help in suggesting the presence of the mitral regurgitation, as Miller, Gibson, and Stephen (1973) have previously indicated.

In the second case the perforation of the left ventricle in the atrioventricular groove became evident as bypass was terminated and the immediate reinstitution of cardiopulmonary bypass, as in Case 1, was an important factor in the satisfactory outcome. The cause of the perforation in this case is uncertain. However, the tissues were noted to be friable and the size $4 \mathrm{M}$ Starr-Edwards prosthesis was a tight fit; this may have led to stretching and tearing the valve ring.

We believe that these two cases have shown that the correct approach to the problem of perforation of the ventricle in the atrioventricular groove is to remove the prosthetic valve and repair the tear from inside. In the two cases reported by Spellberg and O'Reilly (1972) the ventricular perforation and subsequent false aneurysm formation was thought to result from dissection of pericardial adhesions. However, the site of the hole in the left ventricle, in both cases, was very close to the posterior atrioventricular groove, and it seems likely that the mechanism is similar to that described in this report. We recommend that all patients who have repair of a ventricular perforation, particularly if this was in the atrioventricular groove, should have: left ventriculography performed before discharge.

After a mitral valve replacement a pansystolic murmur may not represent mitral regurgitation but may be produced by a false ventricular aneurysm. In addition, mitral regurgitation, with or without a pansystolic murmur, may not be a simple paravalvar leak. We, therefore, recommend left ventriculography when surgery is contemplated, even in the presence of an aortic valve prosthesis, and even though echocardiography indicates mitral regurgitation.

\section{References}

Forrester, J. S., Diamond, G., Freedman, S., Allen, H. N., Parmley, W. W., Matloff, J., and Swann, H. J. C. (1971). 
Silent mitral insufficiency in acute myocardial infarction. Circulation, 44, 877.

Gueron, M., Wanderman, K. L., Hirsch, M., and Borman, J. (1974). Pseudoaneurysms of the left ventricle after myocardial infarction; a curable form of myocardial rupture. Fournal of Thoracic and Cardiovascular Surgery, 69, 736.

Kerr, W. F., Wilcken, D. E. L., and Steiner, R. E. (1961). Incisional aneurysms of the left ventricle. British Heart fournal, 23, 88.

Layton, C., and Hately, W. (1975). Rupture of a left ventricular diverticulum with pseudoaneurysm formation. British Fournal of Radiology, 48, 223.

MacNeil, D. J., Vieweg, W. V. R., Oury, J. H., Folkerth, T. D., and Hagan, A. D. (1974). Pseudomitral regurgitation due to false aneurysm of the left ventricle treated successfully by surgery. Chest, 66, 724 .

MacVaugh, H., IIIrd, Joyner, C. R., and Johnson, J. (1971). Unusual complications during mitral valve replacement in the presence of calcification of the annulus. Annals of Thoracic Surgery, 11, 336.

Miller, H. C., Gibson, D. G., and Stephens, J. D. (1973). Role of echocardiography and phonocardiography in the diagnosis of mitral paraprosthetic regurgitation with Starr-Edwards prostheses. British Heart fournal, 35, 1217.

O'Reilly, R. J., Kazenelson, G., and Spellberg, R. D. (1970). Traumatic pseudoaneurysm of the left ventricle. American fournal of Diseases of Children, 120, 252.
Roberts, W. C., and Morrow, A. G. (1967). Causes of early postoperative death following cardiac valve replacement. fournal of Thoracic and Cardiovascular Surgery, 54, 422.

Sanders, C. A. (1969). Editorial. Prosthetic problems. New England Fournal of Medicine, 281, 501.

Spellberg, R. D., and O'Reilly, R. J. (1972). Pseudoaneurysm of the left ventricle following mitral valve replacement. Chest, 62, 115.

Treasure, R. L., Rainer, W. G., and Sadler, T. R. (1974). Intraoperative left ventricular rupture associated with mitral valve replacement. Chest, 66,511 .

Vlodaver, Z., Coe, J. I., and Edwards, J. E. (1975). True and false left ventricular aneurysms: propensity for the latter to rupture. Circulation, 51, 567.

Wychulis, A. R., Frye, R. L., Kincaid, O. W., Ongley, P. A., and Ellis, F. H., Jr. (1971). Postventriculotomy aneurysm in patients with idiopathic hypertrophic subaortic stenosis. American fournal of Cardiology, 27, 322.

Zacharias, A., Groves, L. K., Cheanvechai, C., Loop, F. D., and Effler, D. B. (1975). Rupture of the posterior wall of the left ventricle after mitral valve replacement. fournal of Thoracic and Cardiovascular Surgery, 69, 259.

Requests for reprints to Dr. G. P. Sharratt, Western Hospital, Oakley. Road, Southampton SO9 4WQ. 